\title{
Fatores associados a quedas em idosos residentes em um bairro de Fortaleza, Ceará
}

\author{
Fall-relatedfactorsin dderly residentsinFortaleza, CearáState, Brazil
}

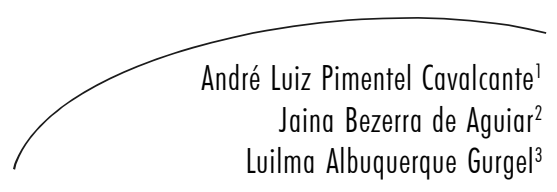

Resumo

A queda é uma das consequências mais graves do envelhecimento, sendo reconhecida como importante problema de saúde pública. A pesquisa teve como objetivo investigar aspectos relacionados à ocorrência de quedas em idosos. Participaram do estudo 50 idosos, do bairro Cidade 2000, em Fortaleza-CE, sendo $64 \%$ do gênero feminino. Para a coleta de dados foi utilizado um questionário. Observou-se que $42 \%$ dos idosos apresentaram, no mínimo, um episódio de queda nos últimos dois anos, dos quais 19\% se encontravam entre 60 e 69 anos, $24 \%$ entre 70 e 79 anos e $57 \%$ entre 80 e 89 anos. As causas foram principalmente relacionadas ao ambiente doméstico inadequado (57\%). Dentre os fatores relacionados ao ambiente doméstico que favorecem as quedas, o mais citado foi a existência de superfícies escorregadias (33\%). A maioria dos participantes $(80 \%)$ fazia uso de algum tipo de medicação prescrita por médicos, sendo os antihipertensivos utilizados por $42 \%$ do grupo. A consequência mais comum das quedas foi a fratura, indicada por $43 \%$ dos idosos, tendo sido mais frequente a fratura de rádio $(56 \%)$. Outras consequências citadas foram trauma craniano (19\%), depressão (19\%) e ansiedade (19\%). Necessitaram de internação hospitalar $33 \%$ dos idosos vítimas de quedas. A pesquisa aponta que $60 \%$ do grupo praticam atividade física regular e, dentre os sedentários, encontravam-se $90 \%$ dos idosos que haviam sido acometidos por quedas. Conclui-se que as quedas podem ser entendidas como eventos que podem, em parte, ser evitados através da adoção de programas e medidas preventivas simples.

\footnotetext{
Curso de Especialização em Saúde do Idoso. Universidade Estadual do Ceará. Fortaleza, CE, Brasil.

2 Curso de Pós-graduação em Saúde Pública. Universidade Estadual do Ceará. Fortaleza, CE, Brasil.

3 Centro de Ciências da Saúde, Universidade Estadual do Ceará. Fortaleza, CE, Brasil.
}

Correspondência / Correspondence

Luilma Albuquerque Gurgel

Centro de Ciências da Saúde, Universidade Estadual do Ceará

Av. Dedé Brasil, $\mathrm{n}^{\circ}$ 1700, Campus do Itaperi

60.740-903 Fortaleza, CE, Brasil

E-mail: luilmagurgel@yahoo.com.br

Palavras-chave: Idoso. Acidentes por Quedas. Saúde do idoso. Causas Externas. 


\section{Abstract}

Fall is one of the most serious consequences of aging, being recognized as an important public health problem. The research aimed to investigate aspects related to occurrence of falls in older adults. Fifty elderly residents of the neighborhood Cidade 2000, in Fortaleza, Ceará state, participated in the study, $64 \%$ were female. For data collection a questionnaire was used. It was observed that $42 \%$ of seniors had at least one fall in the last two years, $19 \%$ of these were between 60 and 69 years, $24 \%$ between 70 and 79 years and $57 \%$ between 80 and 89 years. The causes were mainly related to inadequate home environment $(57 \%)$. Among the factors related to the domestic environment, the most often cited was the existence of slippery surfaces (33\%). Most participants (80\%) used some type of medication prescribed by doctors, and antihypertensive drugs were used by $42 \%$ of the group. The most common consequence of falls was the fracture, indicated by $43 \%$ of elderly, the radius fracture was the most frequent $(56 \%)$. Other consequences mentioned were head trauma (19\%), depression (19\%) and anxiety (19\%); 33\% of elderly victims of falls required hospitalization. The survey found that $60 \%$ of the group reported regular physical activity and $90 \%$ of elderly people who had falls were sedentary. We conclude that falls can be understood as events that may in part be avoided by adopting simple preventive measures and programs.

\author{
Key words: Aged. \\ Accidental Falls. Health of \\ the Elderly External \\ Causes.
}

\section{INTRODUÇÃO}

Com o avançar da idade, ocorre diminuição da eficiência dos sistemas sensoriais (vestibular, visual, somatossensorial). Essa alteração, associada à falta de capacidade para selecionar informações sensoriais de grande importância, pode ser responsável pelo aumento da oscilação corporal e desequilíbrios em idosos, ${ }^{1}$ contribuindo para a ocorrência de queda.

As causas das quedas são multifacetadas, incluindo fatores intrínsecos, comportamentais e ambientais, sendo que a probabilidade para ocorrência de queda aumenta à medida que se acumulam os fatores de risco. ${ }^{2,3}$ Dentre os fatores que vêm sendo responsabilizados pelo aumento do risco de quedas e fraturas na população de idosos, encontra-se o uso de medicamentos que provocam sonolência, alteram o equilíbrio, a tonicidade muscular e/ou provocam hipotensão. ${ }^{4}$

As quedas estão dentre as principais causas de morbidade e mortalidade na população idosa. ${ }^{2}$ Aproximadamente $30 \%$ das pessoas com mais de 65 anos e metade das com mais de 80 anos sofrem uma queda a cada ano. ${ }^{5}$ Segundo estudo realizado no período de 1996 a 2005, as mortes por causas externas, no Brasil, levaram a óbito 41.054 idosos, sendo $22,5 \%(n=9.249)$ codificados como quedas. $^{6}$

As quedas em idosos produzem importante perda de autonomia, prejuízo à qualidade de vida e trazem custos para o sistema de saúde pública, devido principalmente às internações para tratamento de consequência desses acidentes. Além disso, podem ainda repercutir entre seus cuidadores, principalmente os familiares, que devem se mobilizar em torno de cuidados especiais, modificando toda a rotina em função da recuperação ou adaptação após a queda. ${ }^{7}$

Dessa forma, as quedas podem ser consideradas como uma das consequências mais graves do envelhecimento, sendo reconhecidas como importante problema de saúde pública, devido a frequência, morbidade e elevado custo social e econômico decorrente das lesões provocadas. ${ }^{8}$ Isso justifica a realização deste estudo, cujo objetivo foi investigar aspectos relacionados à ocorrência de quedas em idosos, tais como faixa etária e gênero mais acometidos, fatores de risco, associação ao uso de medicamentos, consequências físicas e 
psicológicas, ocorrência de internação hospitalar e associação à prática de exercícios físicos.

Compreender a relação entre a ocorrência de quedas e suas consequências para o idoso favorece o conhecimento da equipe de saúde, o que pode contribuir com a assistência ao cuidado, bem como na proposição de estratégias de promoção da saúde e de prevenção de quedas com vistas à melhoria das condições de vida desses idosos.

\section{METODOLOGIA}

Foi realizado estudo de corte transversal, de caráter descritivo com abordagem quantitativa. Utilizou-se uma amostra composta por 50 idosos, moradores do bairro Cidade 2000, selecionada de forma intencional e por critérios. ${ }^{9}$ A seleção do bairro foi realizada de forma aleatória, por sorteio, entre os bairros de Fortaleza. O local é caracterizado por possuir casas pequenas, bem próximas umas das outras e ruas estreitas chamadas de alamedas, divididas por quadras.

Inicialmente, fixou-se em 400 o número máximo de domicílios a serem visitados. Em seguida, mediante mapeamento, o bairro foi dividido em dez setores e realizou-se uma escolha aleatória, de dez alamedas por setor e de quatro domicílios por alameda. As alamedas foram selecionadas por sorteio e os domicílios selecionados foram os correspondentes às casas de menor e maior número de cada alameda, bem como àquelas casas cujos números correspondiam aos dois quartis intermediários. As visitas foram interrompidas quando se atingiu o número máximo de dois idosos ou quatro domicílios por alameda. Tal critério para composição da amostra foi definido a fim de se obter melhor distribuição das visitas, objetivando alcançar uma amostra representativa do bairro.

Participaram do estudo idosos com idade igual ou superior a 60 anos, de ambos os sexos, não institucionalizados e residentes no bairro Cidade 2000, da cidade de Fortaleza-CE. Consideraramse fatores de exclusão: limitações físicas e sensoriais, acuidade visual e auditiva gravemente diminuídas e absolutamente incapacitantes às atividades de vida diária, amputações, uso de próteses, sequelas neurológicas, doença de Parkinson e impossibilidade de deambular independentemente.

Em 2010, Fortaleza possuía uma população de 2.452.185 habitantes. ${ }^{10} \mathrm{O}$ censo demográfico realizado pelo IBGE em 2000 registrou 160.321 idosos no município e 7.885 habitantes no bairro selecionado para este estudo, sendo 844 idosos. ${ }^{11}$

Foi utilizado como instrumento de coleta de dados um questionário contendo perguntas objetivas, desenvolvido pelos pesquisadores, com o propósito de alcançar os objetivos do estudo. O questionário foi aplicado pelo pesquisador que realizou a leitura de cada uma das perguntas e de suas alternativas de resposta. As entrevistas foram realizadas, nos meses de julho a setembro de 2008, com os idosos em presença de um familiar ou cuidador, que confirmou os dados obtidos ou colaborou, cedendo informações quando necessário.

As variáveis investigadas neste estudo foram: gênero, faixa etária (60-69, 70-79 e 80 anos ou mais), número de episódios de quedas nos últimos 24 meses, causas de acidentes por quedas, fatores relacionados ao ambiente doméstico que favorecem as quedas, consumo de medicamentos, conseqüências físicas e psicológicas das quedas e prática de atividade física. Durante a coleta de dados, foram preenchidos 54 questionários, dos quais quatro foram excluídos da análise dos dados por apresentarem preenchimento incompleto ou inconsistência nos dados.

A análise dos dados foi realizada utilizando-se estatística descritiva, através de distribuição da frequência relativa. Para analisar a relação entre o uso de medicamentos e a ocorrência de quedas, foi aplicado o coeficiente de correlação de Pearson (r). O teste estatístico foi realizado por meio do software SPSS for Windows (Statistical Packeage for Social Sciences, versão 16.0).

O protocolo de pesquisa foi aprovado pelo Comitê de Ética em Pesquisa da Universidade Estadual do Ceará (processo n $\left.{ }^{\circ} 07199442-4\right)$. Os 
participantes demonstraram seu consentimento em participar do estudo através da assinatura de um termo de consentimento livre e esclarecido.

\section{RESULTADOS}

Participaram do estudo 50 pessoas, de ambos os gêneros, com idades entre 60 e 89 anos. Dentre os idosos selecionados para o estudo, $64 \%$ pertenciam ao gênero feminino e $36 \%$, ao masculino. Quanto à faixa etária, 40\%, 28\% e 32\% pertenciam, respectivamente, às faixas etárias de 60-69, 70-79 e 80-89 anos.

Foi verificado que $42 \%$ dos idosos entrevistados apresentaram, no mínimo, um episódio de queda nos últimos dois anos antes da aplicação do questionário, dos quais $71 \%$ eram do gênero feminino. A ocorrência de queda foi verificada em $46,87 \%$ e $33,33 \%$ das mulheres e homens, respectivamente. Quanto ao número de quedas nesse período, $29 \%$ dos idosos sofreram uma queda, $33 \%$ duas e $38 \%$ de três a cinco. A análise foi desagregada por faixas etárias, sendo observado que nas faixas de 60-69, 70-79 e 80-89 anos, a ocorrência de quedas foi relatada por $19 \%$, $24 \%$ e $57 \%$ dos participantes, respectivamente.

Como observado na figura 1 , a maioria das quedas apresenta como causa o ambiente doméstico inadequado. Identificou-se que todos os acidentes ocasionados por quedas ocorreram no próprio lar do idoso, e dentre os fatores relacionados ao ambiente doméstico que favorecem as quedas, o mais citado foi superfície escorregadia (figura 2).

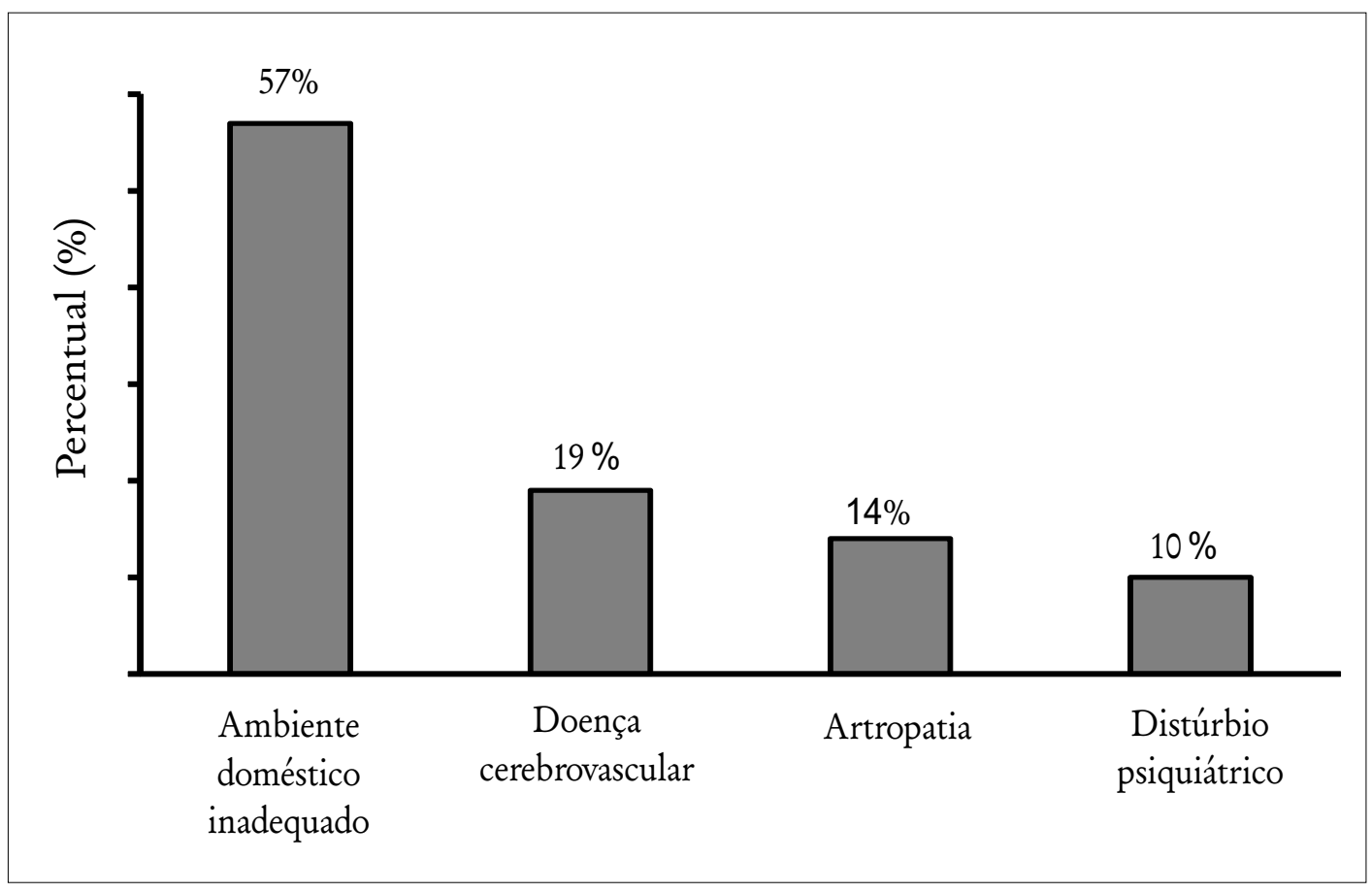

Figura 1 - Distribuição das causas de acidentes por quedas em idosos no bairro Cidade 2000. Fortaleza, CE, 2008. 


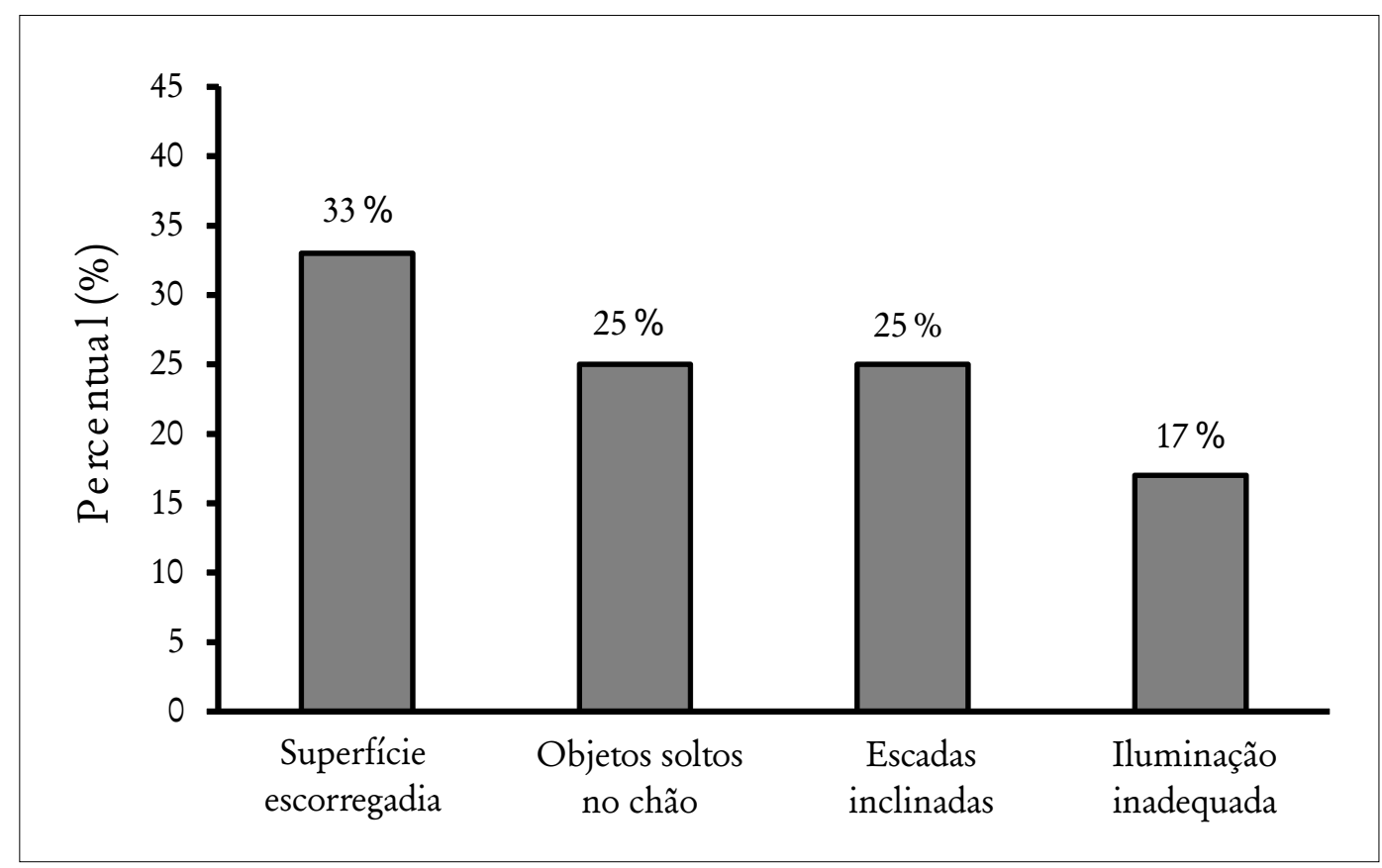

Figura 2 - Distribuição dos fatores relacionados ao ambiente doméstico que favoreceram as quedas em idosos no bairro Cidade 2000. Fortaleza, CE, 2008.

A maioria dos participantes (80\%) fazia uso de algum tipo de medicação prescrita por médicos. $\mathrm{O}$ anti-hipertensivo foi a classe de medicamentos mais consumida, seguida por analgésicos, hipoglicemiantes e antidepressivos. O uso de polifármacos não foi observado entre os idosos (figura 3).

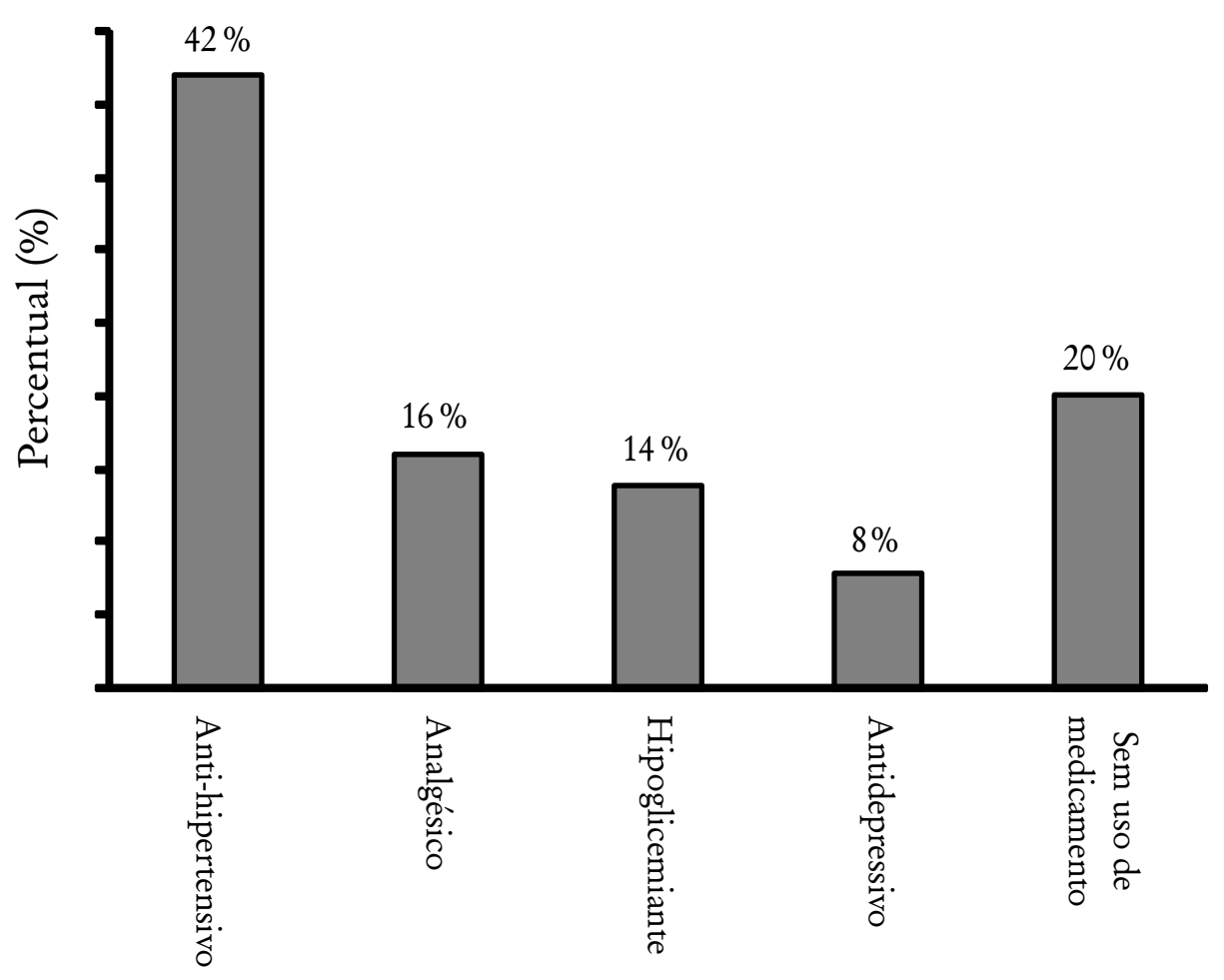

Figura 3 - Distribuição dos idosos de acordo com o consumo de medicamentos, bairro Cidade 2000. Fortaleza, CE, 2008. 
Foram verificadas as consequências físicas e psicológicas após as quedas. A de maior ocorrência foi a fratura, indicada por $43 \%$ dos idosos. Outras consequências citadas foram trauma craniano (19\%), depressão (19\%) e ansiedade (19\%). O local mais frequente das fraturas foi o rádio (56\%), seguido por clavícula $(33 \%)$ e costela $(11 \%)$.

Constatou-se que $33 \%$ dos idosos que caíram foram submetidos à internação hospitalar. Dentre os idosos que estiveram internados, $58 \%$ permaneceram no hospital por até 48 horas para observação médica, em consequência de trauma craniano, e $42 \%$ permaneceram no hospital por período superior a 48 horas para intervenção cirúrgica, em decorrência de fratura.

Dos idosos pesquisados, $40 \%$ eram sedentários e os demais praticavam caminhada $(40 \%)$ ou hidroginástica (20\%), no mínimo duas vezes por semana, há, no mínimo, seis meses. No subgrupo dos sedentários encontravam-se $90 \%$ dos idosos que haviam sido acometidos por quedas.

\section{DISCUSSÃO}

A amostra da presente pesquisa foi composta por idosos com idade mínima de 60 anos, considerando que, de acordo com o Estatuto do Idoso, uma pessoa com idade superior ou igual a 60 anos passa a fazer parte deste grupo. ${ }^{12}$

Os dados encontrados divergem de outras pesquisas que também investigaram a ocorrência de quedas durante 24 meses. Perracini e Ramos encontraram menor percentual de quedas que $o$ verificado neste estudo, $32,7 \%,{ }^{13}$ enquanto Resnick encontrou maior percentual, $77 \% .{ }^{14}$ Assim como Perracini e Ramos, ${ }^{13}$ outros estudiosos também encontraram percentuais inferiores, possivelmente porque estes investigaram a ocorrência que quedas em menor período de tempo, 12 meses. ${ }^{13,15-17}$ Estudo de revisão sistemática realizado por Gama e GómezConesa mostrou que não há consenso na literatura quanto ao período de investigação da ocorrência de quedas, ${ }^{18}$ o que pode dificultar a comparação entre os resultados obtidos pelos diversos estudos.
A prevalência de mulheres na amostra não influenciou a maior ocorrência de quedas observada entre os gerontos do gênero feminino, haja vista que quando os resultados foram analisados por gênero, as mulheres apresentaram maior percentual de ocorrência de quedas quando comparadas com os homens. A maior ocorrência de quedas em mulheres também foi observada em outros estudos. ${ }^{7,8,15}$

Em relação à faixa etária com maior ocorrência de quedas, os dados obtidos corroboram os encontrados em outros estudos, que verificaram maior incidência de quedas entre os idosos mais velhos, ou seja, com 80 anos ou mais em relação àqueles com idade entre 60 e 79 anos. ${ }^{7,19}$ Os resultados confirmam que idosos acima de 80 anos apresentam maior risco relativo de quedas do que idosos de faixa etária inferior. ${ }^{20}$

Em contraste, estudo com 26 idosos da cidade de Marília, São Paulo, verificou que a média de quedas foi maior na faixa etária de 75-84 anos. Segundo os pesquisadores, este fato ocorre provavelmente porque os idosos com 85 anos ou mais diminuem suas atividades naturalmente. ${ }^{21}$

Quanto às causas das quedas, problemas ambientais também foram as mais frequentes causas encontradas em outros estudos. ${ }^{8,22} \mathrm{Em}$ relação ao local de ocorrência das quedas, pesquisas recentes verificaram que a maioria das quedas entre idosos ocorre na própria residência, ${ }^{19,23-25}$ indicando que se trata de eventos relativamente simples, passíveis de serem reduzidos com a adoção de programas e medidas preventivas. Os resultados apontam que todos os acidentes ocorreram na própria residência, o que talvez signifique uma triste realidade frequentemente observada na vida de alguns idosos, por diversos motivos, que saem pouco de casa.

Alguns estudos investigaram fatores relacionados ao ambiente doméstico que favorecem as quedas em idosos, e também encontraram que dentre os fatores de risco mais comuns está o piso escorregadio. ${ }^{8,23}$ 
As pesquisas brasileiras têm dado grande ênfase aos elementos físicos ambientais relacionados às quedas em idosos. Estes dados podem subsidiar a adoção de medidas preventivas de quedas nessa população, como por exemplo, a adaptação da residência do idoso, a fim de oferecer a segurança necessária, minimizando o risco de quedas e suas consequências.

O uso de medicamentos por idosos é frequente e possivelmente crescerá nos próximos anos, em virtude do aumento da expectativa de vida no Brasil. Os resultados encontrados neste estudo se assemelham aos mostrados por Fanhani et al., ${ }^{26}$ que identificaram uso de algum tipo de medicamento por $89 \%$ dos idosos entrevistados. Esses pesquisadores também constataram que os antihipertensivos são os medicamentos mais consumidos.

Estudo de revisão da literatura destacou que os benzodiazepínicos, neurolépticos, antidepressivos e a polimedicação estão associados ao risco aumentado de quedas. ${ }^{18}$ Pesquisa desenvolvida no Brasil também mostrou relação entre o uso de maior número de medicamentos e a ocorrência de quedas. ${ }^{17}$ Esperávamos encontrar uso de polimedicação entre os idosos estudados, mas não foi identificada a utilização concomitante de mais de um fármaco na presente pesquisa, assim como não foi verificada relação entre quedas e uso de medicação $(r=0,1223)$. Tal associação também não foi encontrada em estudo realizado com 50 idosos em internação hospitalar decorrente de quedas. ${ }^{8}$

Quanto ao uso de medicação psicotrópica, este tem sido fator de risco para quedas em indivíduos idosos. Guimarães \& Farinatti encontraram associação entre o uso de medicações psicoativas e risco de queda em mulheres idosas. ${ }^{27}$ No presente estudo, não houve relação entre o uso de antidepressivos e queda, fato que pode ser explicado em virtude de um grande número de idosos estudados não fazer uso deste tipo de medicação, o que também foi observado no estudo desenvolvido por Gai et al. ${ }^{28}$

Tendo em vista a associação comprovada na literatura entre uso de medicamentos e a ocorrência de quedas, ${ }^{18}$ e que estas podem muitas vezes ser causadoras de morte, ${ }^{19}$ faz-se necessário avaliar os possíveis riscos e benefícios dos medicamentos, antes da indicação dos mesmos pelos profissionais de saúde, bem como orientar os idosos e seus familiares quanto aos prejuízos da auto-medicação. Os resultados encontrados assemelham-se aos obtidos por Carvalhaes et al., que citam as fraturas, o trauma craniano, a depressão, o declínio de saúde e a restrição das atividades cotidianas como as consequências mais comuns dos acidentes ocasionados por quedas nos idosos. $^{29}$

$\mathrm{Na}$ presente pesquisa, foi encontrado percentual bastante elevado de fraturas dentre os idosos que sofreram queda, quando comparado com o relatado no estudo de Siqueira et al. ${ }^{17}$ Este achado é preocupante, pois as fraturas decorrentes de quedas são responsáveis por aproximadamente $70 \%$ das mortes acidentais em pessoas acima de 75 anos. $^{30}$

Dentre as fraturas que ocorrem em consequência às quedas, a literatura mostra que são frequentes as de fêmur, ${ }^{23}$ seguidas pelas de rádio. ${ }^{8}$ Entretanto, os idosos entrevistados não relataram a ocorrência de fraturas de fêmur, apesar de as fraturas de rádio terem estado presentes em $56 \%$ dos idosos vítimas de fraturas.

As quedas também podem ter consequências negativas no âmbito psicológico, gerando sintomas como medo e ansiedade diante da possibilidade de uma nova queda. ${ }^{31} \mathrm{O}$ medo de cair pode levar à restrição de mobilidade, diminuição da atividade física, isolamento social e depressão. ${ }^{8,21,32}$

Em estudo desenvolvido por Gawryszewski, ${ }^{19}$ as quedas foram responsáveis pela maior proporção de internações, de atendimentos nas emergências e de mortes por causas definidas. $\mathrm{O}$ tempo médio de internação dos pacientes devido às quedas foi 6,2 dias. ${ }^{19}$ Verificamos que aproximadamente um terço dos idosos vítimas de quedas necessitou de internação hospitalar; destes, $58 \%$ permaneceram internados no máximo por 48 horas. Entretanto, $42 \%$ foram submetidos 
à intervenção cirúrgica e, portanto, tiveram tempo de internação superior.

Assim como na pesquisa realizada por Siqueira et al., os idosos sedentários apresentaram maior prevalência de quedas. ${ }^{17}$ Esses dados estão em coerência com vários estudos que mostram que o exercício físico regular pode melhorar as capacidades funcionais, como a do equilíbrio, da coordenação e da agilidade, ${ }^{33}$ além de minimizar os fatores de risco de quedas de idosos. ${ }^{13} \mathrm{~A}$ prática da atividade física promove estímulos que aprimoram as condições de recepção de informações sensoriais do sistema vestibular, visual e somatossensorial, de modo a ativar os músculos antigravitacionais e estimular o equilíbrio. ${ }^{34}$

O estudo permitiu identificar que as modalidades de exercícios praticadas pelos idosos entrevistados são: a caminhada e a hidroginástica, sendo a primeira mais comum entre os idosos. A caminhada é indicada para idosos por ser considerada atividade leve ou moderada, sendo destaque nos programas de incentivo à prática de atividade física. ${ }^{35} \mathrm{~A}$ hidroginástica também é modalidade bastante recomendada para os idosos, por ser de baixo impacto e melhorar o equilíbrio corporal. ${ }^{36}$

São necessárias ações preventivas na atenção primária à saúde, sobretudo em relação à oferta de atividades físicas que certamente contribuiriam com a redução do risco de quedas. Deve-se reforçar a importância do autocuidado e alertar as famílias e cuidadores para que participem da prevenção de queda com os idosos. Outro aspecto relevante é adaptar a infraestrutura dos domicílios, objetivando reduzir a ocorrência de quedas.

É importante valorizar o evento queda, uma vez que a morte pode ser uma de suas consequências. Minimizar a incidência de quedas em idosos é um desafio para muitas instituições, profissionais e pesquisadores. Para a identificação da intervenção mais apropriada ao alcance deste objetivo, é necessária a realização de estudos que identifiquem os fatores de risco para quedas em idosos.
O estudo apresenta algumas limitações: em primeiro lugar, pode-se questionar a precisão dos dados relatados, uma vez que estes foram obtidos por meio de estratégias recordatórias. $\mathrm{O}$ contato com familiares contribuiu para diminuir o risco de viés, mas ainda assim o problema não pode ser desconsiderado. Além disso, é preciso reconhecer o limitado poder de discriminação da estatística descritiva, que restringe o potencial de generalização das conclusões.

\section{CONCLUSÃO}

O presente trabalho apresentou o perfil dos idosos que sofreram quedas no Bairro Cidade 2000, em Forteleza, Ceará. Os resultados mostram que a faixa etária mais acometida foi a de 80-89 anos; as causas mais frequentes foram as relacionadas aos fatores ambientais, especialmente a existência de pisos escorregadios; os antihipertensivos foram a classe de medicamentos mais utilizados; a fratura configurou-se como a consequência mais comum das quedas, sendo mais frequente as fraturas de rádio; um terço das quedas levou à internação hospitalar e a maioria destas teve duração de até 48 horas. Com relação à prática de atividade física, quase todos os idosos que sofreram quedas eram sedentários.

Em face da importância das quedas do idoso como problema de saúde pública, medidas devem ser tomadas, como cuidados básicos de segurança e prevenção da ocorrência de quedas, em especial nas situações em que elas estão ligadas a fatores ambientais.

A queda pode ser evitada, desde que a equipe de saúde e a família estejam atentas tanto aos fatores intrínsecos como extrínsecos que a predispõem. Dessa forma, espera-se que este estudo possa contribuir para subsidiar a criação e ampliação de políticas públicas, para a prevenção e enfrentamento da ocorrência de quedas em idosos. Recomendase que sejam desenvolvidas novas investigações visando à prevenção de quedas na população idosa, com o intuito de minimizar a repercussão desse evento na capacidade funcional do idoso, contribuindo com o envelhecimento saudável. 


\section{REFERÊNCIAS}

1. Freitas Junior P, Barela JA. Alterações no funcionamento do sistema de controle postural de idosos: uso da informação visual. Rev Port Cien Desp 2006; 6(1): 94-105.

2. Rubenstein LZ. Falls in older people: epidemiology, risk factors and strategies for prevention. Age Ageing 2006; 35(S2): 37-41.

3. Ueno M, et al. Systematic review of fall-related factors among the house-dwelling elderly in Japan. Nippon Ronen Igakkai Zasshi 2006; 43(1): 92-101.

4. Cumming RG, et al. Medications and multiple falls in elderly people: the St. Louis OASIS study. Age Ageing 1991; 20(6): 455-61.

5. Feder G, et al. Guidelines for the prevention of falls in people over 65 . The guidelines' development group. BMJ 2000; 321(7267): 100711.

6. Maciel SSSV, et al. Perfil epidemiológico das quedas em idosos residentes em capitais brasileiras utilizando o sistema de Informações sobre mortalidade. Rev AMRIGS 2010; 54(1): 25-31.

7. Coutinho ES, Silva SD. Uso de medicamentos como fator de risco para fratura grave decorrente de quedas em idosos. Cad Saúde Pública 2002; 18(5): 1359-66.

8. Fabrício SCC, Rodrigues RA, Costa Junior ML. Causas e consequências de quedas de idosos atendidos em hospital público. Rev Saúde Pública 2004; 38(1): 93-9.

9. Patton MQ. Qualitative research \& evaluation methods. 3. ed. London: Sage Publications; 2001. $598 \mathrm{p}$.

10. Instituto Brasileiro de Geografia e Estatística ( IBGE). Sinopse do Censo Demográfico 2010. Disponível em: http://www.ibge.gov.br/home/ estatistica/populacao/censo2010/tabelas_pdf/ Ceara.pdf.

11. Instituto Brasileiro de Geografia e Estatística ( IBGE). Censo Demográfico. 2000. Disponível em: http://www.ibge.gov.br/censo.

12. Brasil. Lei $\mathrm{n}^{\circ} 10.741$ Estatuto do Idoso 1 out 2003. Diário Oficial da União 3 out 2003.

13. Perracine MR, Ramos LR. Fatores associados a quedas em uma coorte de idosos residentes na comunidade. Rev Saúde Pública 2002; 36(6): 709-16.

14. Resnick B. Falls in a community of older adults: putting research into practice. Clin Nurs Res 1999; 8(3): 251-66.
15. Álvares LM, Lima RC, Silva RA. Ocorrência de quedas em idosos residentes em instituições de longa permanência em Pelotas. Cad Saúde Pública 2010; 26(1): 31-40.

16. Carpenter CR, et al. Identification of fall risk factors in older adult emergency department patients. Acad Emerg Med 2009; 16(3): 211-9.

17. Siqueira FV, et al. Prevalência de quedas em idosos e fatores associados. Rev Saúde Pública 2007; 41(5): 749-56.

18. Gama ZAS, Gómez-Conesa A. Factores de riesgo de caídas en ancianos: revisión sistemática. Rev Saúde Pública 2008; 42(5): 94656.

19. Gawryszewski VP. A importância das quedas no mesmo nível entre idosos no estado de São Paulo. Rev Assoc Med Bras 2010; 56(2): 162-7.

20. Barnett A, et al. Community-based group exercise improves balance and reduces falls in at-risk older people: a randomized controlled trial. Age Ageing 2003; 32(4): 407-14.

21. Freitas MAV, Scheicher ME. Preocupação de idosos em relação a quedas. Rev Bras Geriatr Gerontol 2008; 11(1): 57-64.

22. Berg WP, et al. Circunstances and consequences of falls in independent community: dwelling older adults. Age Ageing 1997; 26(4): 261-8.

23. Coutinho ESF, Bloch KV, Rodrigues LC. Characteristics and circumstances of falls leading to severe fractures in elderly people in Rio de Janeiro. Cad Saúde Pública 2009; 25(2): 455-9.

24. Hamra A, Ribeiro MB, Miguel OF. Correlação entre fratura por queda em idosos e uso prévio de medicamentos. Acta Ortop Bras 2007; 15(3): 143-5.

25. Lopes MCL, et al. Fatores desencadeantes de quedas no domicílio em uma comunidade de idosos. Cogitare Enferm 2007; 12(4): 472-7.

26. Fanhani HR, et al. Consumo de medicamentos por idosos atendidos em um centro de convivência no noroeste do Paraná. Rev Bras Geriatr Gerontol 2007; 10(3): 301-14.

27. Guimarães JMN, Farinatti PTV. Análise descritiva de variáveis teoricamente associadas ao risco de quedas em mulheres idosas. Rev Bras Med Esporte 2005; 11(5): 299-305.

28. Gai J, et al. Fatores associados a quedas em mulheres idosas residentes na comunidade. Rev Assoc Med Bras 2010; 56(3): 327-32. 
29. Carvalhaes N, et al. Quedas. In: Anais do 1. Congresso Paulista de Geriatria e Gerontologia;1998 jul 10-14; São Paulo, Brasil. São Paulo: Sociedade Brasileira de Geriatria e Gerontologia; 1998. p. 5-18.

30. Fuller GF. Falls in the elderly. Am Fam Physician 2000; 61(7): 2159-68.

31. Vu MQ, Weintraub N, Rubenstein LZ. Falls in the nursing home: are they preventable? J Am Med Dir Assoc 2004; 5(6): 401-6.

32. Lopes KT, et al. Prevalência do medo de cair em uma população de idosos da comunidade e sua correlação com mobilidade, equilíbrio dinâmico, risco e histórico de quedas. Rev Bras Fisioter 2009; 13(3): 223-9.

33. Silva A, et al. Equilíbrio, coordenação e agilidade de idosos submetidos à prática de exercícios físicos resistidos. Rev Bras Med Esporte 2008; 14(2): 88-93.

34. Masud T, Morris RO. Epidemiology of falls. Age Ageing 2001; 30(S4): 3-7.

35. Hallal PC, et al. Physical inactivity: prevalence and associated variables in Brazilian adults. Med Sci Sports Exerc 2003; 35(11): 1894-900.

36. Mann L, et al. Investigação do equilíbrio corporal em idosos. Rev Bras Geriatr Gerontol 2008; 11(2): 155-65. 